\title{
The Complexity of Reasoning for Fragments of Default Logic*
}

\author{
Olaf Beyersdorff, Arne Meier, Michael Thomas, and Heribert Vollmer \\ Institut für Theoretische Informatik, Gottfried Wilhelm Leibniz Universität \\ Appelstr. 4, 30167 Hannover, Germany \\ \{beyersdorff, meier, thomas, vollmer\}@thi.uni-hannover.de
}

\begin{abstract}
Default logic was introduced by Reiter in 1980. In 1992, Gottlob classified the complexity of the extension existence problem for propositional default logic as $\Sigma_{2}^{\mathrm{p}}$-complete, and the complexity of the credulous and skeptical reasoning problem as $\Sigma_{2}^{\mathrm{p}}$-complete, resp. $\Pi_{2}^{\mathrm{p}}$-complete. Additionally, he investigated restrictions on the default rules, i.e., semi-normal default rules. Selman made in 1992 a similar approach with disjunction-free and unary default rules. In this paper we systematically restrict the set of allowed propositional connectives. We give a complete complexity classification for all sets of Boolean functions in the meaning of Post's lattice for all three common decision problems for propositional default logic. We show that the complexity is a hexachotomy $\left(\Sigma_{2^{-}}^{\mathrm{p}}, \Delta_{2^{-}}^{\mathrm{p}}\right.$, NP-, P-, NL-complete, trivial) for the extension existence problem, while for the credulous and skeptical reasoning problem we obtain similar classifications without trivial cases.
\end{abstract}

Key words: computational complexity, default logic, nonmonotonic reasoning, Post's lattice

\section{Introduction}

When formal specifications are to be verified against real-world situations, one has to overcome the qualification problem that denotes the impossibility of listing all conditions required to decide compliance with the specification. To overcome this problem, McCarthy proposed the introduction of "common-sense" into formal logic [McC80]. Among the formalisms developed since then, Reiter's default logic is one of the best known and most successful formalisms for modeling common-sense reasoning. Default logic extends the usual logical (firstorder or propositional) derivations by patterns for default assumptions. These are of the form "in the absence of contrary information, assume ...". Reiter argued that his logic is an adequate formalization of the human reasoning under the closed world assumption. In fact, today default logic is used in various areas of artificial intelligence and computational logic.

What makes default logic computationally presumably harder than propositional or first-order logic is the fact that the semantics (i.e., the set of consequences) of a given set of premises is defined in terms of a fixed-point equation. The different fixed points (known as extensions or expansions) correspond to different possible sets of knowledge of an agent, based on the given premises.

\footnotetext{
* A preliminary version of this paper appeared in the proceedings of the conference SAT'09 [BMTV09b]. This work was supported by the German Research Foundation (Deutsche Forschungsgemeinschaft) under grants KO 1053/5-2 and VO 630/6-1.
} 
In a seminal paper from 1992, Georg Gottlob classified the complexity of three important decision problems for default logic:

1. Given a set of premises, decide whether it has an extension at all.

2. Given a set of premises and a formula, decide whether the formula occurs in at least one extension (so called brave or credulous reasoning).

3. Given a set of premises and a formula, decide whether the formula occurs in all extensions (cautious or skeptical reasoning).

While in the case of first-order default logic, all these computational tasks are undecidable, Gottlob proved that for propositional default logic, the first and second are complete for the class $\Sigma_{2}^{\mathrm{p}}$, the second level of the polynomial hierarchy (Meyer-Stockmeyer hierarchy), while the third is complete for the class $\Pi_{2}^{\mathrm{p}}$ (the class of complements of $\Sigma_{2}^{\mathrm{p}}$ sets).

In the past, various semantic and syntactic restrictions have been proposed in order to identify computationally easier or even tractable fragments (see, e.g., [Sti90,KS91,BEZ02]). This is the starting point of the present paper. We propose a systematic study of fragments of default logic defined by restricting the set of allowed propositional connectives. For instance, if we look at the fragment where we forbid negation and the constant 0 and allow only conjunction and disjunction, we show that while the first problem is trivial (there always is an extension, in fact a unique one), the second and third problem become coNPcomplete. In this paper we look at all possible sets $B$ of propositional connectives and study the three decision problems defined by Gottlob when all involved formulae contain only connectives from $B$. The computational complexity of the problems then, of course, becomes a function of $B$. We will see that Post's lattice of all closed classes of Boolean functions is the right way to study all such sets $B$. Depending on the location of $B$ in this lattice, we completely classify the complexity of all three reasoning tasks, see Figs. 1 and 2. We will show that, depending on the set $B$ of occurring connectives, the problem of determining the existence of an extension is either $\Sigma_{2}^{\mathrm{p}}$-complete, $\Delta_{2}^{\mathrm{p}}$-complete, NP-complete, P-complete, NL-complete, or trivial, while for the reasoning problems the trivial cases split up into coNP-complete, P-complete, and NL-complete ones (under constant-depth reductions).

The motivation behind our approach lies in the hope that identifying fragments of default logic with simpler reasoning procedures may help us to understand the sources of hardness for the full problem and to locate the boundary between hard and easy fragments. In particular, these procedures may lead to algorithms for solving the studied problems more efficiently.

This paper is organized as follows. After some preliminary remarks in Section 2, we introduce Boolean clones in Section 3. At this place we also provide a full classification of the complexity of logical implications for fragments of propositional logic, as this classification will serve as a central tool for subsequent sections. In Section 4, we start to investigate propositional default logic. Section 5 then presents our main results on the complexity of the decision problems for default logic. Finally, in Section 6 we conclude with a summary and a discussion. 


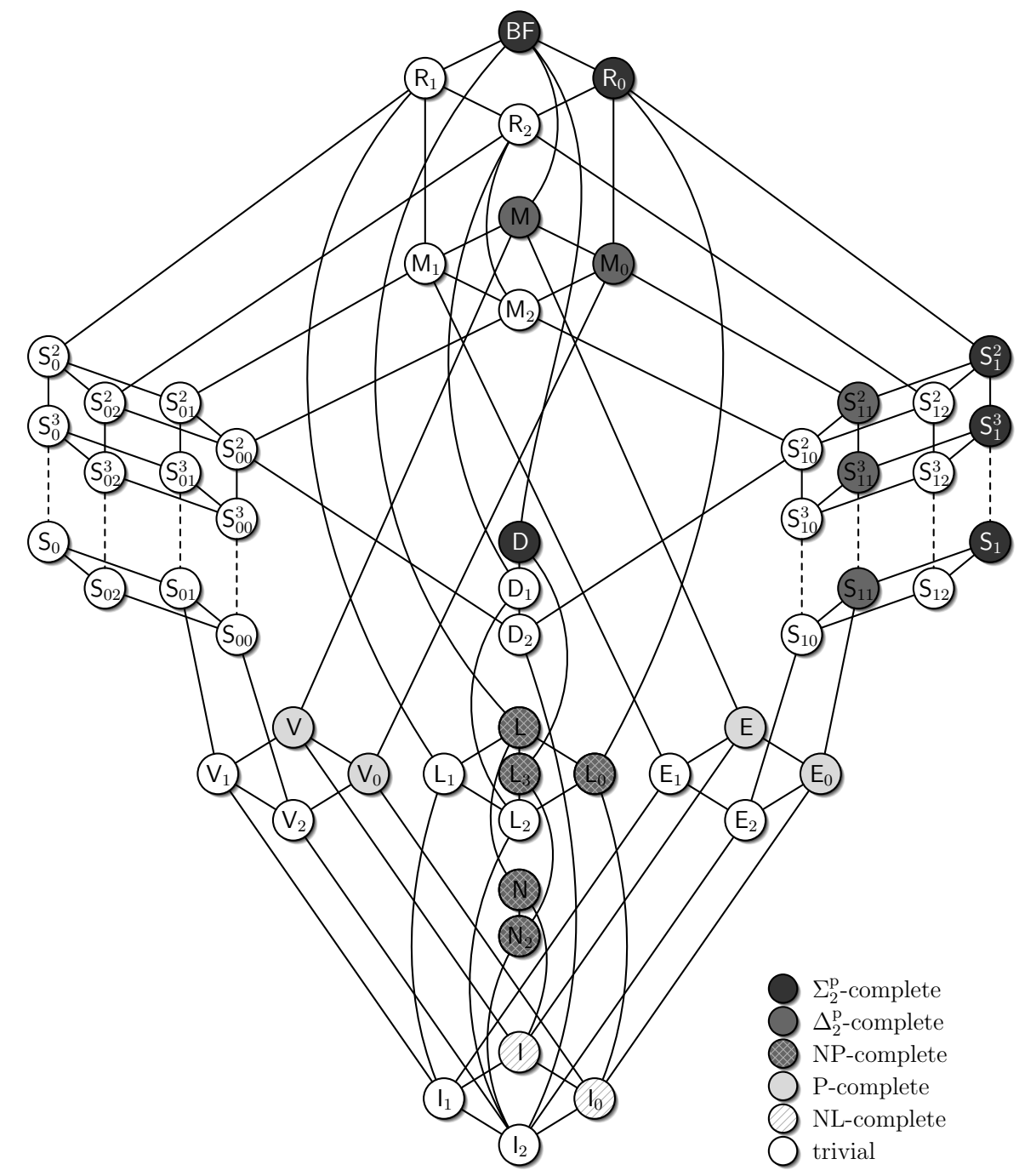

Fig. 1. Post's lattice. Colours indicate the complexity of $\operatorname{EXT}(B)$, the Extension Existence Problem for $B$-formulae.

\section{Preliminaries}

In this paper we make use of standard notions of complexity theory. The arising complexity degrees encompass the classes NL, P, NP, coNP, $\Sigma_{2}^{\mathrm{p}}$ and $\Pi_{2}^{\mathrm{p}}$. For a thorough introduction into the field, the reader is referred to [Pap94]. For the hardness results, we use constant-depth reductions, defined as follows: A language $A$ is constant-depth reducible to a language $B\left(A \leq_{\mathrm{cd}} B\right)$ if there exists a logtime-uniform $\mathrm{AC}^{0}$-circuit family $\left\{C_{n}\right\}_{n \geq 0}$ with unbounded fan-in $\{\wedge, \vee, \neg\}$-gates and oracle gates for $B$ such that for all $x, C_{|x|}(x)=1$ if and only if $x \in A$ (cf. [Vol99]).

We assume familiarity with propositional logic. The set of all propositional formulae is denoted by $\mathcal{L}$. For $A \subseteq \mathcal{L}$ and $\varphi \in \mathcal{L}$, we write $A \models \varphi$ if and only if all assignments satisfying all formulae in $A$ also satisfy $\varphi$. By $\operatorname{Th}(A)$ we denote the set of all consequences of $A$, i.e., $\operatorname{Th}(A)=\{\varphi \mid A \models \varphi\}$. For a literal $\ell$ and 


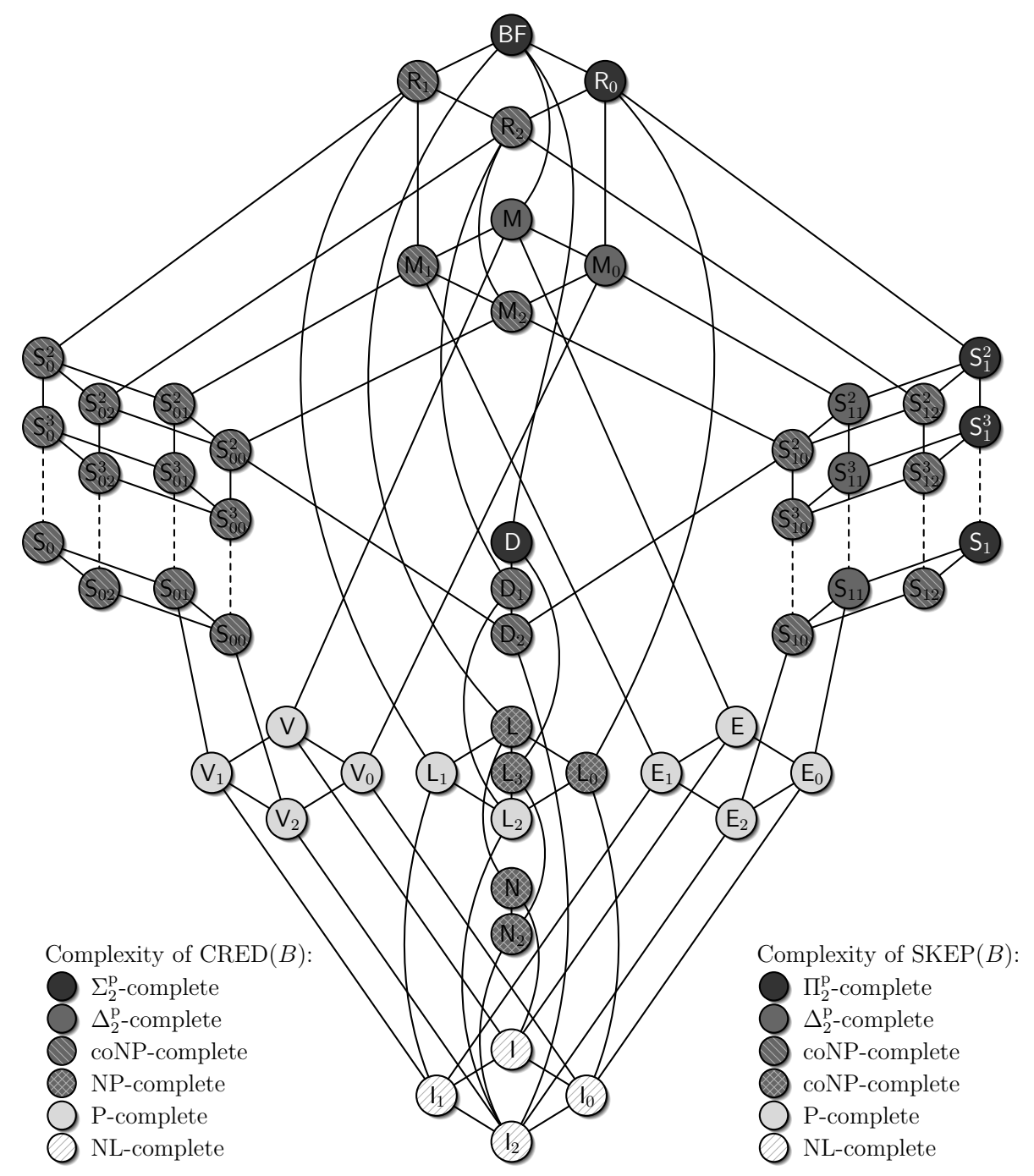

Fig. 2. Post's lattice. Colours indicate the complexity of $\operatorname{CRED}(B)$ and $\operatorname{SKEP}(B)$, the Credulous and Skeptical Reasoning Problems for $B$-formulae.

a variable $x$, we define $\bar{\ell}$ as the literal of opposite polarity, i.e., $\bar{\ell}:=x$ if $\ell=\neg x$ and $\bar{\ell}:=\neg x$ if $\ell=x$. For a formula $\varphi$, let $\varphi_{[\alpha / \beta]}$ denote $\varphi$ with all occurrences of the formula $\alpha$ replaced by the formula $\beta$, and let $A_{[\alpha / \beta]}:=\left\{\varphi_{[\alpha / \beta]} \mid \varphi \in A\right\}$ for $A \subseteq \mathcal{L}$.

\section{Boolean Clones and the Complexity of the Implication Problem}

A propositional formula using only connectives from a finite set $B$ of Boolean functions is called a $B$-formula. The set of all $B$-formulae is denoted by $\mathcal{L}(B)$. In order to cope with the infinitely many finite sets $B$ of Boolean functions, we require some algebraic tools to classify the complexity of the infinitely many arising reasoning problems. A clone is a set $B$ of Boolean functions that is closed under superposition, i.e., $B$ contains all projections and is closed under 


\begin{tabular}{|c|c|c|}
\hline Name & Definition & Base \\
\hline $\mathrm{BF}$ & All Boolean functions & $\{\wedge, \neg\}$ \\
\hline $\mathrm{R}_{0}$ & $\{f: f$ is 0 -reproducing $\}$ & $\{\wedge, \not \rightarrow\}$ \\
\hline $\mathrm{R}_{1}$ & $\{f: f$ is 1-reproducing $\}$ & $\{\vee, \rightarrow\}$ \\
\hline M & $\{f: f$ is monotone $\}$ & $\{\vee, \wedge, 0,1\}$ \\
\hline $\mathrm{S}_{0}$ & $\{f: f$ is 0 -separating $\}$ & $\{\rightarrow\}$ \\
\hline $\mathrm{S}_{1}$ & $\{f: f$ is 1 -separating $\}$ & $\{\not \rightarrow\}$ \\
\hline $\mathrm{S}_{00}$ & $\mathrm{~S}_{0} \cap \mathrm{R}_{0} \cap \mathrm{R}_{1} \cap \mathrm{M}$ & $\{x \vee(y \wedge z)\}$ \\
\hline $\mathrm{S}_{10}$ & $S_{1} \cap R_{0} \cap R_{1} \cap M$ & $\{x \wedge(y \vee z)\}$ \\
\hline $\mathrm{S}_{11}$ & $\mathrm{~S}_{1} \cap \mathrm{M}$ & $\{x \wedge(y \vee z), 0\}$ \\
\hline $\mathrm{D}$ & $\{f: f$ is self-dual $\}$ & $\{(x \wedge \bar{y}) \vee(x \wedge \bar{z}) \vee(\bar{y} \wedge \bar{z})\}$ \\
\hline $\mathrm{D}_{2}$ & $\mathrm{D} \cap \mathrm{M}$ & $\{(x \wedge y) \vee(y \wedge z) \vee(x \wedge z)\}$ \\
\hline $\mathrm{L}$ & $\{f: f$ is linear $\}$ & $\{\oplus, 1\}$ \\
\hline $\mathrm{L}_{0}$ & $L \cap R_{0}$ & $\{\oplus\}$ \\
\hline $\mathrm{L}_{1}$ & $L \cap R_{1}$ & $\{\equiv\}$ \\
\hline $\mathrm{L}_{2}$ & $L \cap R_{0} \cap R_{1}$ & $\{x \oplus y \oplus z\}$ \\
\hline $\mathrm{L}_{3}$ & $L \cap D$ & $\{x \oplus y \oplus z, \neg\}$ \\
\hline $\mathrm{V}$ & $\left\{f: f \equiv c_{0} \vee \bigvee_{i=1}^{n} c_{i} x_{i}\right.$ where the $c_{i}$ s are constant $\}$ & $\{\vee, 0,1\}$ \\
\hline $\mathrm{V}_{2}$ & {$[\{\vee\}]$} & $\{\vee\}$ \\
\hline $\mathrm{E}$ & $\left\{f: f \equiv c_{0} \wedge \bigwedge_{i=1}^{n} c_{i} x_{i}\right.$ where the $c_{i}$ s are constant $\}$ & $\{\wedge, 0,1\}$ \\
\hline$E_{2}$ & {$[\{\wedge\}]$} & $\{\wedge\}$ \\
\hline $\mathrm{N}$ & $\{f: f$ depends on at most one variable $\}$ & $\{\neg, 0,1\}$ \\
\hline $\mathrm{N}_{2}$ & {$[\{\neg\}]$} & $\{\neg\}$ \\
\hline 1 & $\{f: f$ is a projection or a constant $\}$ & $\{$ id, 0,1$\}$ \\
\hline $\mathrm{I}_{2}$ & {$[\{i d\}]$} & $\{\mathrm{id}\}$ \\
\hline
\end{tabular}

Table 1. A list of Boolean clones with definitions and bases.

arbitrary composition [Pip97, Chapter 1]. For an arbitrary set $B$ of Boolean functions, we denote by $[B]$ the smallest clone containing $B$ and call $B$ a base for $[B]$. In [Pos41] Post classified the lattice of all clones and found a finite base for each clone, see Fig. 1. In order to introduce the clones relevant to this paper, we define the following notions for $n$-ary Boolean functions $f$ :

- $f$ is c-reproducing if $f(c, \ldots, c)=c, c \in\{0,1\}$.

- $f$ is monotone if $a_{1} \leq b_{1}, a_{2} \leq b_{2}, \ldots, a_{n} \leq b_{n}$ implies $f\left(a_{1}, \ldots, a_{n}\right) \leq$ $f\left(b_{1}, \ldots, b_{n}\right)$.

- $f$ is $c$-separating if there exists an $i \in\{1, \ldots, n\}$ such that $f\left(a_{1}, \ldots, a_{n}\right)=c$ implies $a_{i}=c, c \in\{0,1\}$.

$-f$ is self-dual if $f \equiv \operatorname{dual}(f)$, where $\operatorname{dual}(f)\left(x_{1}, \ldots, x_{n}\right)=\neg f\left(\neg x_{1}, \ldots, \neg x_{n}\right)$.

- $f$ is linear if $f \equiv x_{1} \oplus \cdots \oplus x_{n} \oplus c$ for a constant $c \in\{0,1\}$ and variables $x_{1}, \ldots, x_{n}$.

The clones relevant to this paper are listed in Table 1. The definition of all Boolean clones can be found, e.g., in [BCRV03].

For a finite set $B$ of Boolean functions, we define the Implication Problem for $B$-formulae $\operatorname{IMP}(B)$ as the following computational task: Given a set $A$ of $B$-formulae and a $B$-formula $\varphi$, decide whether $A \models \varphi$ holds. The complexity of the implication problem is classified in [BMTV09a]. The results relevant to this paper are summarized in the following theorem.

Theorem 3.1 ([BMTV09a, Theorem 4.1]). Let B be a finite set of Boolean functions. Then $\operatorname{IMP}(B)$ is 
1. coNP-complete if $\mathrm{S}_{00} \subseteq[B], \mathrm{S}_{10} \subseteq[B]$ or $\mathrm{D}_{2} \subseteq[B]$, and

2. in $\mathrm{P}$ for all other cases.

\section{Default Logic}

Fix some finite set $B$ of Boolean functions and let $\alpha, \beta, \gamma$ be propositional $B$ formulae. A $B$-default (rule) is an expression $d=\frac{\alpha: \beta}{\gamma} ; \alpha$ is called prerequisite, $\beta$ is called justification and $\gamma$ is called consequent of $d$. A $B$-default theory is a pair $\langle W, D\rangle$, where $W$ is a set of propositional $B$-formulae and $D$ is a finite set of $B$-default rules. Henceforth we will omit the prefix " $B$-" if $B=\mathrm{BF}$ or the meaning is clear from the context.

For a given default theory $\langle W, D\rangle$ and a set of formulae $E$, let $\Gamma(E)$ be the smallest set of formulae such that

1. $W \subseteq \Gamma(E)$,

2. $\Gamma(E)$ is closed under deduction, i.e., $\Gamma(E)=\operatorname{Th}(\Gamma(E))$, and

3. for all defaults $\frac{\alpha: \beta}{\gamma} \in D$ with $\alpha \in \Gamma(E)$ and $\neg \beta \notin E$, it holds that $\gamma \in \Gamma(E)$.

A (stable) extension of $\langle W, D\rangle$ is a fix-point of $\Gamma$, i.e., a set $E$ such that $E=$ $\Gamma(E)$.

The following theorem by Reiter provides an alternative characterization of extensions:

Theorem 4.1 ([Rei80]). Let $\langle W, D\rangle$ be a default theory and $E$ be a set of formulae.

1. Let $E_{0}=W$ and $E_{i+1}=\operatorname{Th}\left(E_{i}\right) \cup\left\{\gamma \mid \frac{\alpha: \beta}{\gamma} \in D, \alpha \in E_{i}\right.$ and $\left.\neg \beta \notin E\right\}$. Then $E$ is a stable extension of $\langle W, D\rangle$ if and only if $E=\bigcup_{i \in \mathbb{N}} E_{i}$.

2. Let $G=\left\{\frac{\alpha: \beta}{\gamma} \in D \mid \alpha \in E\right.$ and $\left.\neg \beta \notin E\right\}$. If $E$ is a stable extension of $\langle W, D\rangle$, then

$$
E=\operatorname{Th}\left(W \cup\left\{\gamma \mid \frac{\alpha: \beta}{\gamma} \in G\right\}\right) .
$$

In this case, $G$ is also called the set of generating defaults for $E$.

Observe that, as an immediate consequence of Theorem 4.1, stable extensions possess polynomial-sized witnesses, namely the set of their generating defaults. Moreover, note that stable extensions need not be consistent. However, the following proposition shows that this only occurs if the set $W$ is already inconsistent.

Proposition 4.2 ([MT93, Corollary 3.60]). Let $\langle W, D\rangle$ be a default theory. Then $\mathcal{L}$ is a stable extension of $\langle W, D\rangle$ if and only if $W$ is inconsistent.

As a consequence we obtain:

Corollary 4.3. Let $\langle W, D\rangle$ be a default theory.

- If $W$ is consistent, then every stable extension of $\langle W, D\rangle$ is consistent.

- If $W$ is inconsistent, then $\langle W, D\rangle$ has a stable extension.

The main reasoning tasks in nonmonotonic logics give rise to the following three decision problems: 
1. the Extension Existence Problem $\operatorname{EXT}(B)$

Instance: a $B$-default theory $\langle W, D\rangle$

Question: Does $\langle W, D\rangle$ have a stable extension?

2. the Credulous Reasoning Problem $\operatorname{CRED}(B)$

Instance: a $B$-formula $\varphi$ and a $B$-default theory $\langle W, D\rangle$

Question: Is there a stable extension of $\langle W, D\rangle$ that includes $\varphi$ ?

3. the Skeptical Reasoning Problem $\operatorname{SKEP}(B)$

Instance: a $B$-formula $\varphi$ and a $B$-default theory $\langle W, D\rangle$

Question: Does every stable extension of $\langle W, D\rangle$ include $\varphi$ ?

The next theorem follows from [Got92] and states the complexity of the above decision problems for the general case $[B]=\mathrm{BF}$.

Theorem 4.4. Let $B$ be a finite set of Boolean functions such that $[B]=$ $\mathrm{BF}$. Then $\operatorname{EXT}(B)$ and $\operatorname{CRED}(B)$ are $\Sigma_{2}^{\mathrm{p}}$-complete, whereas $\operatorname{SKEP}(B)$ is $\Pi_{2}^{\mathrm{p}}$ complete.

Proof. The upper bounds given in [Got92] do not depend on the Boolean connectives allowed and thus hold for any finite set $B$ of Boolean functions. For $\Sigma_{2}^{\mathrm{p}}$ - and $\Pi_{2}^{\mathrm{p}}$-hardness, it suffices to note that if $[B]=\mathrm{BF}$, then there exist $B$ formulae $f(x, y), g(x, y)$ and $h(x)$ such that $f(x, y) \equiv x \wedge y, g(x, y) \equiv x \vee y$, $h(x) \equiv \neg x$ and both $x$ and $y$ occur at most once in $f, g$, and $h$ [Lew79]. Hence, the hardness results generalize to arbitrary bases $B$ with $[B]=\mathrm{BF}$.

\section{The Complexity of Default Reasoning}

In this section we will classify the complexity of the three problems $\operatorname{EXT}(B)$, $\operatorname{CRED}(B)$, and $\operatorname{SKEP}(B)$ for all choices of Boolean connectives $B$. We start with some preparations which will substantially reduce the number of cases we have to consider.

Lemma 5.1. Let $\mathrm{P}$ be any of the problems EXT, CRED, or SKEP. Then for each finite set $B$ of Boolean functions, $\mathrm{P}(B) \equiv_{\mathrm{cd}} \mathrm{P}(B \cup\{1\})$.

Proof. The reductions $\mathrm{P}(B) \leq_{\mathrm{cd}} \mathrm{P}(B \cup\{1\})$ are obvious. For the converse reductions, we will essentially substitute the constant 1 by a new variable $t$ that is forced to be true (this trick goes back to Lewis [Lew79]). For EXT, the reduction is given by $\langle W, D\rangle \mapsto\left\langle W^{\prime}, D^{\prime}\right\rangle$, where $W^{\prime}=W_{[1 / t]} \cup\{t\}, D^{\prime}=D_{[1 / t]}$, and $t$ is a new variable not occurring in $\langle W, D\rangle$. If $\left\langle W^{\prime}, D^{\prime}\right\rangle$ possesses a stable extension $E^{\prime}$, then $t \in E^{\prime}$. Hence, $E_{[t / 1]}^{\prime}$ is a stable extension of $\langle W, D\rangle$. On the other hand, if $E$ is a stable extension of $\langle W, D\rangle$, then $\operatorname{Th}\left(E_{[1 / t]} \cup\{t\}\right)=E_{[1 / t]}$ is a stable extension of $\left\langle W^{\prime}, D^{\prime}\right\rangle$. Therefore, each extension $E$ of $\langle W, D\rangle$ corresponds to the extension $E_{[1 / t]}$ of $\left\langle W^{\prime}, D^{\prime}\right\rangle$, and vice versa.

For the problems CRED and SKEP, it suffices to note that the above reduction $\langle W, D\rangle \mapsto\left\langle W^{\prime}, D^{\prime}\right\rangle$ has the additional property that for each formula $\varphi$ and each extension $E$ of $\langle W, D\rangle, \varphi \in E$ if and only if $\varphi_{[1 / t]} \in E_{[1 / t]}$.

The next lemma shows that, quite often, $B$-default theories have unique extensions. 
Lemma 5.2. Let $B$ be a finite set of Boolean functions. Let $\langle W, D\rangle$ be a $B$ default theory. If $[B] \subseteq \mathrm{R}_{1}$ then $\langle W, D\rangle$ has a unique stable extension. If $[B] \subseteq \mathrm{M}$ then $\langle W, D\rangle$ has at most one stable extension.

Proof. For $[B] \subseteq \mathrm{R}_{1}$, every premise, justification and consequent is 1-reproducing. As all consequences of 1-reproducing functions are again 1-reproducing and the negation of a 1-reproducing function is not 1-reproducing, the justifications in $D$ become irrelevant. Hence the characterization of stable extensions from the first item in Theorem 4.1 simplifies to the following iterative construction: $E_{0}=W$ and $E_{i+1}=\operatorname{Th}\left(E_{i}\right) \cup\left\{\gamma \mid \frac{\alpha: \beta}{\gamma} \in D, \alpha \in E_{i}\right\}$. As $D$ is finite, this construction terminates after finitely many steps, i.e., $E_{k}=E_{k+1}$ for some $k \geq 0$. Then $E=\bigcup_{i<k} E_{i}$ is the unique stable extension of $\langle W, D\rangle$. For a similar result confer [BÖ02, Theorem 4.6].

For $[B] \subseteq \mathrm{M}$, every formula is either 1-reproducing or equivalent to 0 . As rules with justification equivalent to 0 are never applicable, each $B$-default theory $\langle W, D\rangle$ with finite $D$ has at most one stable extension by the same argument as above.

As an immediate corollary, the credulous and the skeptical reasoning problem are equivalent for the above choices of the underlying connectives.

Corollary 5.3. Let $B$ be a finite set of Boolean functions such that $[B] \subseteq \mathrm{R}_{1}$ or $[B] \subseteq \mathrm{M}$. Then $\operatorname{CRED}(B) \equiv_{\mathrm{cd}} \operatorname{SKEP}(B)$.

\subsection{The Extension Existence Problem}

Now we are ready to classify the complexity of EXT. The next theorem shows that this is a hexachotomy: the $\Sigma_{2}^{\mathrm{p}}$-completeness of the general case [Got92] is inherited by all clones above $S_{1}$ and $D$; for monotone sets of connectives the complexity drops to $\Delta_{2}^{\mathrm{p}}$-completeness if $\wedge, \vee$ and 0 are available, and membership in $\mathrm{P}$ otherwise (with this case splitting up into P-completeness, NL-completeness and triviality); lastly, for affine sets of connectives containing $\neg$ or 0 the complexity of EXT reduces to NP-completeness.

Theorem 5.4. Let $B$ be a finite set of Boolean functions. Then $\operatorname{EXT}(B)$ is

1. $\Sigma_{2}^{\mathrm{p}}$-complete if $\mathrm{S}_{1} \subseteq[B] \subseteq \mathrm{BF}$ or $\mathrm{D} \subseteq[B] \subseteq \mathrm{BF}$,

2. $\Delta_{2}^{\mathrm{p}}$-complete if $\mathrm{S}_{11} \subseteq[B] \subseteq \mathrm{M}$,

3. $\mathrm{NP}$-complete if $[B] \in\left\{\mathrm{N}, \mathrm{N}_{2}, \mathrm{~L}, \mathrm{~L}_{0}, \mathrm{~L}_{3}\right\}$,

4. P-complete if $[B] \in\left\{\mathrm{E}, \mathrm{E}_{0}, \mathrm{~V}, \mathrm{~V}_{0}\right\}$,

5. NL-complete if $[B] \in\left\{\mathrm{I}, \mathrm{I}_{0}\right\}$, and

6. trivial in all other cases (i.e., if $[B] \subseteq \mathrm{R}_{1}$ ).

The proof of Theorem 5.4 will be established from the lemmas in this subsection.

Lemma 5.5. Let $B$ be a finite set of Boolean functions such that $\mathrm{S}_{11} \subseteq[B] \subseteq$ M. Then $\operatorname{EXT}(B)$ is $\Delta_{2}^{\mathrm{p}}$-complete. 


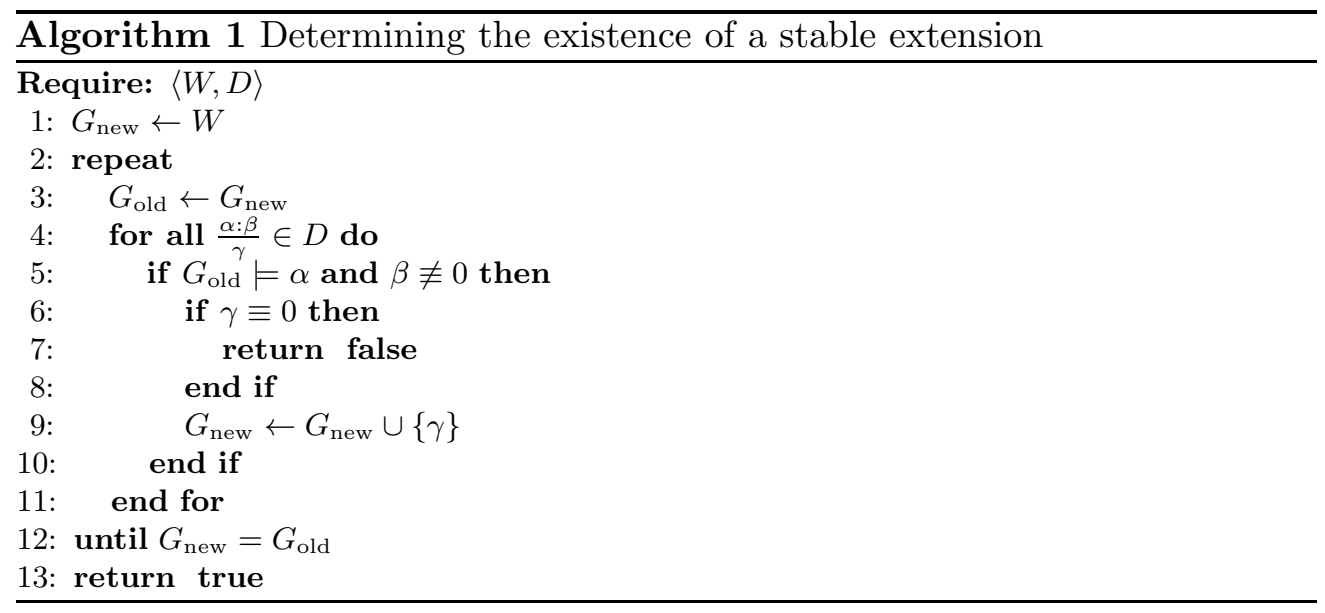

Proof. We start by showing $\operatorname{EXT}(B) \in \Delta_{2}^{\mathrm{p}}$. Let $B$ be a finite set of Boolean functions such that $[B] \subseteq \mathrm{M}$ and $\langle W, D\rangle$ be a $B$-default theory. As the negated justification $\neg \beta$ of every default rule $\frac{\alpha: \beta}{\gamma} \in D$ is either equivalent to the constant 1 or not 1-reproducing, it holds that in the former case $\neg \beta$ is contained in any stable extension, whereas in the latter $\neg \beta$ cannot be contained in a consistent stable extension of $\langle W, D\rangle$. We can distinguish between those two cases in polynomial time. Therefore, using the characterization of Theorem 4.1(1), we can iteratively compute the applicable defaults and test whether the premise of any default with unsatisfiable conclusion can be derived. Algorithm 1 implements these steps on a deterministic Turing machine using a coNP-oracle to test for implication of $B$-formulae. Clearly, Algorithm 1 terminates after a polynomial number of steps. Hence, $\operatorname{EXT}(B)$ is contained in $\Delta_{2}^{\mathrm{p}}$.

To show the $\Delta_{2}^{\mathrm{p}}$-hardness of $\operatorname{EXT}(B)$, we reduce from the $\Delta_{2}^{\mathrm{p}}$-complete problem SNSAT [Got95a, Theorem 3.4] defined as follows:

Problem: SNSAT

Input: A sequence $\left(\varphi^{i}\right)_{1 \leq i \leq n}$ of formulae such that $\varphi^{i}$ contains the propositions $x_{1}, \ldots, x_{i-1}$ and $z_{i 1}, \ldots, z_{i m_{i}}$

Output: Is $c_{n}=1$, where $c_{i}$ is recursively defined via $c_{i}:=1$ if and only if $\varphi^{i}$ is satisfiable by an assignment $\sigma$ such that $\sigma\left(x_{j}\right)=c_{j}$ for all $1 \leq j<i$ ?

Let $\left(\varphi^{i}\right)_{1 \leq i \leq n}$ be the given sequence of propositional formulae and assume without loss of generality that $\varphi^{i}$ is in conjunctive normal form for all $1 \leq i \leq n$. For every proposition $x_{j}$ or $z_{i j}$ occurring in $\left(\varphi^{i}\right)_{1 \leq i \leq n}$, let $x_{j}^{\prime}$ respectively $z_{i j}^{\prime}$ be a fresh proposition, and define

$$
\psi^{i}:=\varphi_{\left[\neg x_{1} / x_{1}^{\prime}, \ldots, \neg x_{i-1} / x_{i-1}^{\prime}, \neg z_{i 1} / z_{i 1}^{\prime}, \ldots, \neg z_{i m_{i}} / z_{i m_{i}}^{\prime}\right]}^{i-1} \wedge \bigwedge_{j=1}^{i-1}\left(x_{j} \vee x_{j}^{\prime}\right) \wedge \bigwedge_{j=1}^{m_{i}}\left(z_{i j} \vee z_{i j}^{\prime}\right) .
$$

The key observation in the relationship of $\varphi^{i}$ and $\psi^{i}$ is that, for all $c_{1}, \ldots, c_{i-1} \in$ $\{0,1\}, \varphi_{\left[x_{1} / c_{1}, \ldots, x_{i-1} / c_{i-1}\right]}^{i}$ is unsatisfiable if and only if for each model $\sigma$ of $\psi_{\left[x_{1} / c_{1}, \ldots, x_{i-1} / c_{i-1}, x_{1}^{\prime} / \neg c_{1}, \ldots, x_{i-1}^{\prime} / \neg c_{i-1}\right]}^{i}$ there exists an index $1 \leq j \leq m_{i}$ such that 
$\sigma$ sets to 1 both $z_{i j}$ and $z_{i j}^{\prime}$. We will use this observation to show that the $B$-default theory $\langle W, D\rangle$ defined below has a stable extension if and only if $\left(\varphi^{i}\right)_{1 \leq i \leq n}$ is an instance of SNSAT, that is, $\varphi_{\left[x_{1} / c_{1}, \ldots, x_{i-1} / c_{i-1}\right]}^{n}$ is satisfiable for $c_{1}, \ldots, c_{i-1}$ recursively defined via

$$
c_{i}:=1 \Longleftrightarrow \varphi_{\left[x_{1} / c_{1}, \ldots, x_{i-1} / c_{i-1}\right]}^{i} \text { is satisfiable. }
$$

Define $W:=\left\{\psi^{1}, \ldots, \psi^{n}\right\}$ and

$$
\begin{aligned}
D:= & \left\{\frac{\bigvee_{j=1}^{m_{i}}\left(z_{i j} \wedge z_{i j}^{\prime}\right) \vee \bigvee_{j=1}^{i-1}\left(x_{j} \wedge x_{j}^{\prime}\right): 1}{x_{i}^{\prime}} \mid 1 \leq i<n\right\} \cup \\
& \left\{\frac{\bigvee_{j=1}^{m_{n}}\left(z_{n j} \wedge z_{n j}^{\prime}\right) \vee \bigvee_{j=1}^{n-1}\left(x_{j} \wedge x_{j}^{\prime}\right): 1}{0}\right\} .
\end{aligned}
$$

We will prove the claim appealing to the characterization of stable extensions from Theorem 4.1 (1). Let $E_{0}:=W$. If $\varphi^{1}$ is unsatisfiable then $\frac{\bigvee_{j=1}^{m_{1}}\left(z_{1 j} \wedge z_{1 j}^{\prime}\right): 1}{x_{1}^{\prime}}$ is applicable and thus $x_{1}^{\prime}$ is added to $E_{1}$. On the other hand, if $\varphi^{1}$ is satisfiable then there exists a model $\sigma$ of $\varphi^{1}$. Define $\hat{\sigma}$ as the extension of $\sigma$ defined as $\hat{\sigma}\left(z_{1 j}^{\prime}\right)=\neg \sigma\left(z_{1 j}\right)$ for all $1 \leq j \leq m_{1}$. By virtue of $\sigma \models \varphi^{1}$ and the construction of $\hat{\sigma}$, we obtain that $\hat{\sigma} \models \psi^{1}$ while $\hat{\sigma} \not \models \bigvee_{j=1}^{m_{1}}\left(z_{1 j} \wedge z_{1 j}^{\prime}\right)$. Summarizing, $\varphi^{1}$ is unsatisfiable if and only if $\frac{\bigvee_{j=1}^{m_{1}}\left(z_{1 j} \wedge z_{1 j}^{\prime}\right): 1}{x_{1}^{\prime}}$ is applicable.

Now suppose that $E_{i}$ is such that for all $j<i$ the proposition $x_{j}^{\prime}$ is contained in $E_{i}$ if and only if $\varphi_{\left[x_{1} / c_{1}, \ldots, x_{j-1} / c_{j-1}\right]}^{j}$ with $c_{1}, \ldots, c_{j-1}$ defined as in (1) is unsatisfiable. If $\varphi_{\left[x_{1} / c_{1}, \ldots, x_{i-1} / c_{i-1}\right]}^{i}$ is unsatisfiable then any model of the formula

$$
\psi^{i} \wedge \bigwedge_{\substack{1 \leq j<i, \sigma\left(c_{j}\right)=1}} x_{j} \wedge \bigwedge_{\substack{1 \leq j<i, \sigma\left(c_{j}\right)=0}} x_{j}^{\prime}
$$

sets to 1 both $z_{i j}$ and $z_{i j}^{\prime}$ for some $1 \leq j \leq m_{i}$. From (2) and the monotonicity of $\psi^{i}$, we obtain that for each model $\sigma^{\prime}$ of $\psi^{i} \wedge \bigwedge_{1 \leq j<i, \sigma\left(c_{j}\right)=0} x_{j}^{\prime}$ there must exist either an index $1 \leq j<i$ such that $\sigma^{\prime}$ sets $x_{j}$ and $x_{j}^{\prime}$ to 1 , or an index $1 \leq j \leq m_{i}$ such that $\sigma^{\prime}$ sets $z_{i j}$ and $z_{i j}^{\prime}$ to 1 . Consequently, $\frac{\bigvee_{j=1}^{m_{i}}\left(z_{i j} \wedge z_{i j}^{\prime}\right) \vee \bigvee_{j=1}^{i-1}\left(x_{j} \wedge x_{j}^{\prime}\right): 1}{x_{i}^{\prime}}$ is applicable and $x_{i}^{\prime} \in E_{i+1}$. On the other hand, if $\varphi_{\left[x_{1} / c_{1}, \ldots, x_{i-1} / c_{i-1}\right]}^{i}$ is satisfiable then there exists a model $\sigma$ that can be extended to $\hat{\sigma}$ by $\hat{\sigma}\left(z_{i j}^{\prime}\right)=\neg \sigma\left(z_{i j}\right)$ for all $1 \leq j \leq m_{i}$ and $\hat{\sigma}\left(x_{j}^{\prime}\right)=\neg \sigma\left(x_{j}\right)$ for all $1 \leq j<i$ such that $\hat{\sigma} \models \psi^{i}$ and $\hat{\sigma} \not \models \bigvee_{j=1}^{m_{i}}\left(z_{i j} \wedge z_{i j}^{\prime}\right) \vee \bigvee_{j=1}^{i-1}\left(x_{j} \wedge x_{j}^{\prime}\right)$. Summarizing, $\varphi^{i}$ is unsatisfiable if and only if $\frac{\bigvee_{j=1}^{m_{i}}\left(z_{i j} \wedge z_{i j}^{\prime}\right) \vee \bigvee_{j=1}^{i-1}\left(x_{j} \wedge x_{j}^{\prime}\right): 1}{x_{i}^{\prime}}$ is applicable.

The direction from right to left now follows from the fact that $\varphi_{n}$ is satisfiable if and only if $\frac{\bigvee_{j=1}^{m_{n}}\left(z_{i j} \wedge z_{i j}^{\prime}\right) \bigvee \bigvee_{j=1}^{n-1}\left(x_{i} \wedge x_{i}^{\prime}\right): 1}{0}$ is not applicable, which in turn implies that $\langle W, D\rangle$ has a stable extension. Conversely, if $\varphi_{\left[x_{1} / c_{1}, \ldots, x_{n-1} / c_{n-1}\right]}^{n}$ is unsatisfiable with $c_{1}, \ldots, c_{n-1}$ defined as in (1), then any model of $\psi^{i} \wedge$ $\bigwedge_{1 \leq j<i, \sigma\left(c_{j}\right)=0} x_{j}^{\prime}$ sets to true either $x_{j}$ and $x_{j}^{\prime}$ for some $1 \leq j<i$ or $z_{i j}$ and 
$z_{i j}^{\prime}$ for some $1 \leq j \leq m_{i}$. As a result, the default $\frac{\bigvee_{j=1}^{m_{n}}\left(z_{i j} \wedge z_{i j}^{\prime}\right) \vee \bigvee_{j=1}^{n-1}\left(x_{j} \wedge x_{j}^{\prime}\right): 1}{0}$ is applicable and $\langle W, D\rangle$ does not possess a stable extension.

Finally, observe that all formulae contained in $\langle W, D\rangle$ are monotone. Hence, $\langle W, D\rangle$ is a $\{\wedge, \vee, 0,1\}$-default theory. Let $B$ be a finite set of Boolean functions such that $S_{11} \subseteq[B]$. Replacing all occurrences of $x \wedge y$ and $x \vee y$ in $\langle W, D\rangle$ with their respective $(B \cup\{1\})$-representations $f_{\wedge}(x, y)$ and $f_{\vee}(x, y)$, and eliminating the constant 1 as in the proof of Lemma 5.1 yields a $B$-default theory $\left\langle W^{B}, D^{B}\right\rangle$ that is equivalent to $\langle W, D\rangle$. The variables $x$ or $y$ may occur several times in the body of $f_{\wedge}$ or $f_{\vee}$, hence $\left\langle W^{B}, D^{B}\right\rangle$ might be exponential in the length of the original input. To avoid this blowup, we exploit the associativity of $\wedge$ and $\vee$ : we insert parentheses such that the conjunctions and disjunctions in each of the above formulae are transformed into trees of logarithmic depth.

Thus we have established a reduction from SNSAT to $\operatorname{EXT}(B)$ for all $B$ such that $\mathrm{S}_{11} \subseteq[B]$. This concludes the proof.

Lemma 5.6. Let $B$ be a finite set of Boolean functions such that $[B] \in\left\{\mathrm{N}, \mathrm{N}_{2}\right.$, $\left.\mathrm{L}, \mathrm{L}_{0}, \mathrm{~L}_{3}\right\}$. Then $\operatorname{EXT}(B)$ is $\mathrm{NP}$-complete.

Proof. We start by showing $\operatorname{EXT}(B) \in \mathrm{NP}$ for $[B] \subseteq \mathrm{L}$. Given a default theory $\langle W, D\rangle$, we first guess a set $G \subseteq D$ which will serve as the set of generating defaults for a stable extension. Let $G^{\prime}=W \cup\left\{\gamma \mid \frac{\alpha: \beta}{\gamma} \in G\right\}$. We use Theorem 4.1 to verify whether $\operatorname{Th}\left(G^{\prime}\right)$ is indeed a stable extension of $\langle W, D\rangle$. For this we inductively compute generators $G_{i}$ for the sets $E_{i}$ from Theorem 4.1, until eventually $E_{i}=E_{i+1}$ (note, that because $D$ is finite, this always occurs). We start by setting $G_{0}=W$. Given $G_{i}$, we check for each rule $\frac{\alpha: \beta}{\gamma} \in D$, whether $G_{i} \models \alpha$ and $G^{\prime} \forall \neg \beta$ (as all formulae belong to $\mathcal{L}(B)$, this is possible by Theorem 3.1). If so, then $\gamma$ is put into $G_{i+1}$. If this process terminates, i.e., if $G_{i}=G_{i+1}$, then we check whether $G^{\prime}=G_{i}$. By Theorem 4.1, this test is positive if and only if $G$ generates a stable extension of $\langle W, D\rangle$.

To show NP-hardness of $\operatorname{EXT}(B)$ for $\mathrm{N} \subseteq[B]$, we will $\leq_{\mathrm{cd}}$-reduce $3 \mathrm{SAT}$ to $\operatorname{EXT}(B)$. Let $\varphi=\bigwedge_{i=1}^{n}\left(\ell_{i 1} \vee \ell_{i 2} \vee \ell_{i 3}\right)$ and $\ell_{i j}$ be literals over propositions $\left\{x_{1}, \ldots, x_{m}\right\}$ for $1 \leq i \leq n, 1 \leq j \leq 3$. We transform $\varphi$ to the $B$-default theory $\left\langle W, D_{\varphi}\right\rangle$, where $W:=\emptyset$ and

$$
\begin{aligned}
D_{\varphi}:= & \left\{\frac{1: x_{i}}{x_{i}} \mid 1 \leq i \leq m\right\} \cup\left\{\frac{1: \neg x_{i}}{\neg x_{i}} \mid 1 \leq i \leq m\right\} \cup \\
& \left\{\frac{\bar{\ell}_{i \pi(1)}: \bar{\ell}_{i \pi(2)}}{\ell_{i \pi(3)}} \mid 1 \leq i \leq n, \pi \text { is a permutation of }\{1,2,3\}\right\} .
\end{aligned}
$$

To prove the correctness of the reduction, first assume $\varphi$ to be satisfiable. For each satisfying assignment $\sigma:\left\{x_{1}, \ldots, x_{m}\right\} \rightarrow\{0,1\}$ for $\varphi$, we claim that

$$
E:=\operatorname{Th}\left(\left\{x_{i} \mid \sigma\left(x_{i}\right)=1\right\} \cup\left\{\neg x_{i} \mid \sigma\left(x_{i}\right)=0\right\}\right)
$$

is a stable extension of $\left\langle W, D_{\varphi}\right\rangle$. We will verify this claim with the help of the first part of Theorem 4.1. Starting with $E_{0}=\emptyset$, we already get $E_{1}=E$ by the default rules $\frac{1: x_{i}}{x_{i}}$ and $\frac{1: \neg x_{i}}{\neg x_{i}}$ in $D_{\varphi}$. As $\sigma$ is a satisfying assignment for $\varphi$, each 
consequent of a default rule in $D_{\varphi}$ is already in $E$. Hence $E_{2}=E_{1}$ and therefore $E=\bigcup_{i \in \mathbb{N}} E_{i}$ is a stable extension of $\left\langle W, D_{\varphi}\right\rangle$.

Conversely, assume that $E$ is a stable extension of $\left\langle W, D_{\varphi}\right\rangle$. Because of the default rules $\frac{1: x_{i}}{x_{i}}$ and $\frac{1: \neg x_{i}}{\neg x_{i}}$, we either get $x_{i} \in E$ or $\neg x_{i} \in E$ for all $i=1, \ldots, m$. The rules of the type $\frac{\bar{\ell}_{i 1} \bar{\ell}_{i 2}}{\ell_{i 3}}$ ensure that $E$ contains at least one literal from each clause $\ell_{i 1} \vee \ell_{i 2} \vee \ell_{i 3}$ in $\varphi$. As $E$ is deductively closed, $E$ contains $\varphi$. By Corollary 4.3, the extension $E$ is consistent, and therefore $\varphi$ is satisfiable.

Hence, $\operatorname{EXT}(B)$ is NP-complete for every finite set $B$ such that $\mathrm{N} \subseteq[B] \subseteq$ $\mathrm{L}$. The remaining cases $[B] \in\left\{\mathrm{N}_{2}, \mathrm{~L}_{0}, \mathrm{~L}_{3}\right\}$ follow from Lemma 5.1, because $\left[\mathrm{N}_{2} \cup\{1\}\right]=\mathrm{N},\left[\mathrm{L}_{0} \cup\{1\}\right]=\mathrm{L}$, and $\left[\mathrm{L}_{3} \cup\{1\}\right]=\mathrm{L}$.

Lemma 5.7. Let $B$ be a finite set of Boolean functions such that $[B] \in\left\{\mathrm{E}_{1} \mathrm{E}_{0}\right.$, $\left.\mathrm{V}, \mathrm{V}_{0}\right\}$. Then $\operatorname{EXT}(B)$ is $\mathrm{P}$-complete.

Proof. Let $B$ be a finite set of Boolean functions such that $[B] \in\left\{\mathrm{E}, \mathrm{E}_{0}, \mathrm{~V}, \mathrm{~V}_{0}\right\}$. Membership in $\mathrm{P}$ is is obtained from Algorithm 1, as for these types of $B$ formulae, we have an efficient test for implication.

To prove P-hardness for $\mathrm{E}_{0} \subseteq[B]$, we provide a reduction from the complement of the accessibility problem for directed hypergraphs, $\overline{\text { HGAP }}$. In directed hypergraphs $H=(V, F)$, hyperedges $e \in F$ consist of a set of source nodes $\operatorname{src}(e) \subseteq V$ and a destination $\operatorname{dest}(e) \in V$. Instances of HGAP contain a directed hypergraph $H=(V, F)$, a set $S \subseteq V$ of source nodes, and a target node $t \in V$. HGAP is P-complete under $\leq_{\mathrm{cd}}$-reductions [SI90], even if restricted to hypergraphs whose edges contain at most two source nodes.

We transform a given instance $(H, S, t)$ to the $\operatorname{EXT}(\{\wedge, 0,1\})$-instance $\langle W, D\rangle$ with

$$
W:=\left\{p_{s} \mid s \in S\right\}, \quad D:=\left\{\frac{\bigwedge_{v \in \operatorname{src}(e)} p_{v}: 1}{p_{\operatorname{dest}(e)}} \mid e \in F\right\} \cup\left\{\frac{p_{t}: 1}{0}\right\}
$$

with pairwise distinct propositions $p_{v}$ for $v \in V$. It is easy to verify that $(H, s, t) \in \mathrm{HGAP} \Longleftrightarrow\langle W, D\rangle \notin \operatorname{EXT}(\{\wedge, 0,1\})$. Using Lemma 5.1 and replacing $\wedge$ by its $B$-representation, we obtain $\overline{\mathrm{HGAP}} \leq_{\mathrm{cd}} \operatorname{EXT}(B)$ for all finite sets $B$ such that $\mathrm{E}_{0} \subseteq[B]$. As $\mathrm{P}$ is closed under complementation, $\operatorname{EXT}(B)$ is P-complete.

$$
\begin{aligned}
& \text { For } \bigvee_{0} \subseteq[B], \text { set } \\
& W:=\left\{\bigvee_{s \notin S} p_{s}\right\}, D:=\left\{\frac{\bigvee_{v \in V \backslash \operatorname{src}(e)} p_{v}: 1}{\bigvee_{v \in V \backslash(\operatorname{src}(e) \cup\{\operatorname{dest}(e)\})} p_{v}} \mid e \in F\right\} \cup\left\{\frac{\bigvee_{v \in V \backslash\{t\}} p_{v}: 1}{0}\right\} .
\end{aligned}
$$

We claim that this mapping realizes the reduction $\overline{\operatorname{HGAP}} \leq_{\text {cd }} \operatorname{EXT}(\{\vee, 0,1\})$. First suppose that $t$ can be reached from $S$ in $H$. Then there exists a sequence $\left(S_{i}\right)_{0 \leq i \leq n}$ of sets of nodes such that $S_{0}=S, t \in S_{n}$, and for all $0 \leq i<n, S_{i+1}$ is obtained from $S_{i}$ by adding the destination $\operatorname{dest}(e)$ of a hyperedge $e \in F$ with $\operatorname{src}(e) \subseteq S_{i}$. Let $\left(e_{i}\right)_{0 \leq i<n}$ denote the corresponding sequence of hyperedges used to obtain $S_{i+1}$ from $S_{i}$. Then, for all $0 \leq i<n$, the following holds:

$$
\operatorname{src}\left(e_{i}\right) \subseteq S_{i} \Longleftrightarrow \frac{\bigvee_{v \in V \backslash \operatorname{src}(e)} p_{v}: 1}{\bigvee_{v \in V \backslash(\operatorname{src}(e) \cup\{\operatorname{dest}(e)\})} p_{v}} \text { is applicable in } E_{i}
$$


where $\left(E_{i}\right)_{i \in \mathbb{N}}$ is the sequence from Theorem $4.1(1)$. As $\bigcup_{i \in \mathbb{N}} E_{i}$ is guaranteed to be unique by Lemma 5.2 and $t \in S_{n}$, we obtain that $0 \in E_{n+1}$. Consequently, $\langle W, D\rangle$ does not possess a stable extension.

Conversely, if $\langle W, D\rangle$ does not admit a stable extension, then 0 has to be derivable. Accordingly, there exists a sequence of defaults $\left(d_{i}\right)_{0 \leq i \leq n}$ such that the premise of $d_{i}$ can be derived from $W \cup\left\{\gamma \mid d_{j}=\frac{\alpha: \beta}{\gamma}, 0 \leq j<i\right\}$ and $d_{n}=\frac{\bigvee_{v \in V \backslash\{t\}} p_{v}: 1}{0}$. By construction of $\langle W, D\rangle$, this sequence can be translated into a sequence $\left(S_{i}\right)_{0 \leq i \leq n}$ of node sets in the hypergraph such that $S_{0}=S$, $t \in S_{n}$, and for all $0 \leq i<n, S_{i+1}$ is obtained from $S_{i}$ by adding the destination $\operatorname{dest}(e)$ of a hyperedge $e \in F$ with $\operatorname{src}(e) \subseteq S_{i}$. Consequently, $t$ is reachable from $S$ in $H$ and we conclude that $\overline{\mathrm{HGAP}} \leq_{\mathrm{cd}} \operatorname{EXT}(\{\vee, 0,1\})$. Using Lemma 5.1, we get $\overline{\mathrm{HGAP}} \leq_{\mathrm{cd}} \operatorname{EXT}(\{\vee, 0\})$.

To see that $\operatorname{EXT}(\{\vee, 0\}) \leq_{\text {cd }} \operatorname{EXT}(B)$ for all finite sets $B$ such that $\mathrm{V}_{0} \subseteq[B]$, we proceed as in the proof of Lemma 5.5 and insert parentheses such that the disjunctions in each of the above formulae are transformed into tree of logarithmic depth. Hence, replacing all occurrences of $\vee$ in $W, D$ and $\varphi$ with its $B$-representation yields an $\operatorname{EXT}(B)$-instance of size polynomial in the original input. Concluding, $\operatorname{EXT}(B)$ is P-complete.

Lemma 5.8. Let $B$ be a finite set of Boolean functions such that $[B] \in\left\{\mathrm{I}, \mathrm{I}_{0}\right\}$. Then $\operatorname{EXT}(B)$ is NL-complete with respect to constant-depth reductions.

Proof. Let $B$ be a finite set of Boolean functions such that $[B] \in\left\{\mathrm{I}, \mathrm{I}_{0}\right\}$. We will first show membership in NL by giving a reduction to the complement of the graph accessibility problem, $\overline{\mathrm{GAP}}$.

Let $\langle W, D\rangle$ be a $B$-default theory. Analogously to the proof of Lemma 5.5, it holds that $\langle W, D\rangle$ has a stable extension if and only if either $W$ is inconsistent or the conclusions of all applicable defaults are consistent. Assume that $W$ is consistent and denote by $D^{\prime} \subseteq D$ those defaults $\frac{\alpha: \beta}{\gamma} \in D$ with $\beta \not \equiv 0$. Then a $B$ default rule $\frac{\alpha: \beta}{\gamma} \in D^{\prime}$ is applicable if and only if the proposition $\alpha$ is contained in $W$ or itself the conclusion of an applicable default. Therefore, testing whether the conclusions of all applicable defaults are consistent is essentially equivalent to solving a reachability problem in a directed graph. Define $G_{\langle W, D\rangle}$ as the directed graph $(V, F)$ with

$$
\begin{aligned}
& V:=\{0,1\} \cup W \cup\left\{\alpha, \gamma \mid \frac{\alpha: \beta}{\gamma} \in D\right\} \\
& F:=\{(1, x) \mid x \in W\} \cup\left\{(\alpha, \gamma) \mid \frac{\alpha: \beta}{\gamma} \in D, \beta \not \equiv 0\right\}
\end{aligned}
$$

if $W$ is consistent, and

$$
\begin{aligned}
& V:=\{0,1\}, \\
& F:=\emptyset
\end{aligned}
$$

otherwise. It is easy to see that $\langle W, D\rangle$ has a stable extension if and only if there is no path from 1 to 0 in $G_{\langle W, D\rangle}$. Thus the function mapping the given $B$-default theory $\langle W, D\rangle$ to the GAP-instance $\left(G_{\langle W, D\rangle}, 1,0\right)$ constitutes a reduction from $\operatorname{EXT}(B)$ to $\overline{\mathrm{GAP}}$. As the consistency of $W$ can be determined in $\mathrm{AC}^{0}$, the 
reduction can be computed using constant-depth circuits. Membership in NL follows from the closure of NL under complementation.

To show NL-hardness, we establish a constant-depth reduction in the converse direction. For a directed graph $G=(V, F)$ and two nodes $s, t \in V$, we transform the given GAP-instance $(G, s, t)$ to $\langle W, D\rangle$ with

$$
W:=\left\{p_{s}\right\}, \quad D:=\left\{\frac{p_{u}: p_{u}}{p_{v}} \mid(u, v) \in F\right\} \cup\left\{\frac{p_{t}: p_{t}}{0}\right\}
$$

Clearly, $(G, s, t) \in \mathrm{GAP}$ if and only if $\langle W, D\rangle$ does not have a stable extension. As NL is closed under complementation, the lemma is established.

Proof (Theorem 5.4). For $\mathrm{S}_{1} \subseteq[B] \subseteq \mathrm{BF}$ or $[B]=\mathrm{D}$, observe that in both cases $\mathrm{BF}=[B \cup\{1\}]$. Claim 1 then follows from Theorem 4.4 and Lemma 5.1. Claims two to five are established in Lemmas 5.5-5.8. For all sets $B$ not captured by the above, it now holds that $[B] \subseteq \mathrm{R}_{1}$. Thus, the sixth claim follow directly from Lemmas 5.2,

\subsection{The Credulous and the Skeptical Reasoning Problem}

We will now analyse the credulous and the skeptical reasoning problems. For these problems, there are two sources of complexity. On the one hand, we need to determine a candidate for a stable extension. On the other hand, we have to verify that this candidate is indeed a finite characterization of some stable extension that includes a given formula - a task that requires to test for formula implication. Depending on the Boolean connectives allowed, one or both tasks can be performed in polynomial time. We obtain the full complexity, i.e., $\Sigma_{2}^{\mathrm{p}}$-completeness for $\operatorname{CRED}(B)$ and $\Pi_{2}^{\mathrm{p}}$-completeness for $\operatorname{SKEP}(B)$, where both problems $\operatorname{EXT}(B)$ and $\operatorname{IMP}(B)$ attain their highest complexity. The complexity reduces to $\Delta_{2}^{\mathrm{p}}$ for clones that allow for an efficient computation of stable extensions but whose implication problem remains coNP-complete. More precisely, the problem is $\Delta_{2}^{\mathrm{p}}$-complete if $0 \in[B]$ and becomes coNP-complete otherwise. Conversely, if the implication problem becomes easy but determining an extension candidate is hard, then $\operatorname{CRED}(B)$ is $\operatorname{NP}$-complete, while $\operatorname{SKEP}(B)$ is coNP-complete. This is the case for $[B] \in\left\{\mathrm{N}, \mathrm{N}_{2}, \mathrm{~L}, \mathrm{~L}_{0}, \mathrm{~L}_{3}\right\}$. Finally, for clones $B$ that allow for solving both tasks in polynomial time, both $\operatorname{CRED}(B)$ and $\operatorname{SKEP}(B)$ are in $\mathrm{P}$.

The complete classification of $\operatorname{CRED}(B)$ is given in the following theorem.

Theorem 5.9. Let $B$ be a finite set of Boolean functions. Then $\operatorname{CRED}(B)$ is

1. $\Sigma_{2}^{\mathrm{p}}$-complete if $\mathrm{S}_{1} \subseteq[B] \subseteq \mathrm{BF}$ or $\mathrm{D} \subseteq[B] \subseteq \mathrm{BF}$,

2. $\Delta_{2}^{\mathrm{p}}$-complete if $\mathrm{S}_{11} \subseteq[B] \subseteq \mathrm{M}$,

3. coNP-complete if $X \subseteq[B] \subseteq \mathrm{R}_{1}$ for $X \in\left\{\mathrm{S}_{00}, \mathrm{~S}_{10}, \mathrm{D}_{2}\right\}$,

4. NP-complete if $[B] \in\left\{\mathrm{N}, \mathrm{N}_{2}, \mathrm{~L}, \mathrm{~L}_{0}, \mathrm{~L}_{3}\right\}$,

5. $\mathrm{P}$-complete if $\mathrm{V}_{2} \subseteq[B] \subseteq \mathrm{V}, \mathrm{E}_{2} \subseteq[B] \subseteq \mathrm{E}$ or $[B] \in\left\{\mathrm{L}_{1}, \mathrm{~L}_{2}\right\}$, and

6. NL-complete if $\mathrm{I}_{2} \subseteq[B] \subseteq \mathrm{I}$.

The proof of Theorem 5.9 follows from the upper and lower bounds given in Propositions 5.10 and 5.11 below. 
Proposition 5.10. Let $B$ be a finite set of Boolean functions. Then $\operatorname{CRED}(B)$ is contained

1. in $\Sigma_{2}^{\mathrm{p}}$ if $\mathrm{S}_{1} \subseteq[B] \subseteq \mathrm{BF}$ or $\mathrm{D} \subseteq[B] \subseteq \mathrm{BF}$,

2. in $\Delta_{2}^{\mathrm{p}}$ if $[B] \subseteq \mathrm{M}$,

3. in coNP if $[B] \subseteq \mathrm{R}_{1}$,

4. in NP if $[B] \subseteq \mathrm{L}$,

5. in $\mathrm{P}$ if $[B] \subseteq \mathrm{V},[B] \subseteq \mathrm{E}$ or $[B] \subseteq \mathrm{L}_{1}$, and

6. in $\mathrm{NL}$ if $[B] \subseteq \mathrm{I}$.

Proof. Part one follows from Theorem 4.4 and Lemma 5.1.

For $[B] \subseteq \mathrm{M}$, membership in $\Delta_{2}^{\mathrm{p}}$ is obtained from a straightforward extension of Algorithm 1: first iteratively compute the applicable defaults $G$ while asserting that $\langle W, D\rangle$ has a stable extension using Algorithm 1, and eventually verify that $\varphi$ is implied by $W$ and the conclusions in $G$.

For $[B] \subseteq \mathrm{R}_{1}$, the justifications $\beta$ are irrelevant for computing a stable extension, as for every default rule $\frac{\alpha: \beta}{\gamma} \in D$ we cannot derive $\neg \beta$ ( $\neg \beta$ is not 1-reproducing). Hence, a unique consistent stable extension $E$ is guaranteed to exist by Theorem 5.2. Using Algorithm 1 we can iteratively compute the generating defaults of $E$ of the unique consistent stable extension of $\langle W, D\rangle$ and eventually check whether $\varphi$ is implied by $W$ and the conclusions in of the generating defaults of $E$.

For $[B] \subseteq \mathrm{L}$, we proceed similarly as in the proof of part 3 in Theorem 5.4. First, we guess a set $G$ of generating defaults and subsequently verify that both $\operatorname{Th}\left(W \cup\left\{\gamma \mid \frac{\alpha: \beta}{\gamma} \in G\right\}\right)$ is a stable extension and that $W \cup\left\{\gamma \mid \frac{\alpha: \beta}{\gamma} \in G\right\} \models \varphi$. Using Theorem 3.1, both conditions may be verified in polynomial time.

For $[B] \subseteq \mathrm{V},[B] \subseteq \mathrm{E}$, and $[B] \subseteq \mathrm{L}_{1}$, we again use Algorithm 1. As for these types of $B$-formulae we have an efficient test for implication (Theorem 3.1), we get $\operatorname{CRED}(B) \in \mathrm{P}$.

For $[B] \subseteq \mathrm{I}$, observe that NL is closed under intersection. Hence, given a $B$-default theory $\langle W, D\rangle$ and a $B$-formula $\varphi$ we can first test whether $\langle W, D\rangle$ has a stable extension $E$ using Lemma 5.8 and subsequently assert that $\varphi \in E$ by reusing the graph $G_{\langle W, D\rangle}$ constructed from $\langle W, D\rangle$ : it holds that $\varphi \in E$ if and only if the node corresponding to $\varphi$ is contained in $G_{\langle W, D\rangle}$ and reachable from the node 1 . Thus, $\operatorname{CRED}(B) \in \mathrm{NL}$.

We will now establish the lower bounds required to complete the proof of Theorem 5.9.

Proposition 5.11. Let $B$ be a finite set of Boolean functions. Then $\operatorname{CRED}(B)$ is

1. $\Sigma_{2}^{\mathrm{p}}$-hard if $\mathrm{S}_{1} \subseteq[B]$ or $\mathrm{D} \subseteq[B]$,

2. $\Delta_{2}^{\mathrm{p}}$-hard if $\mathrm{S}_{11} \subseteq[B]$,

3. coNP-hard if $\mathrm{S}_{00} \subseteq[B], \mathrm{S}_{10} \subseteq[B]$ or $\mathrm{D}_{2} \subseteq[B]$,

4. NP-hard if $\mathrm{N}_{2} \subseteq[B]$ or $\mathrm{L}_{0} \subseteq[B]$,

5. $\mathrm{P}$-hard if $\mathrm{V}_{2} \subseteq[B], \mathrm{E}_{2} \subseteq[B]$ or $\mathrm{L}_{2} \subseteq[B]$, and

6. NL-hard for all other clones. 
Proof. Part one follows from Theorem 4.4 and Lemma 5.1.

For the second part, observe that the constant 1 is contained in any stable extension. The second part thus follows from Lemmas 5.1 and 5.5.

For $\mathrm{S}_{00} \subseteq[B], \mathrm{S}_{10} \subseteq[B]$, and $\mathrm{D}_{2} \subseteq[B]$, coNP-hardness is established by a $\leq_{\text {cd }}$-reduction from $\operatorname{IMP}(B)$. Let $A \subseteq \mathcal{L}(B)$ and $\varphi \in \mathcal{L}(B)$. Then the default theory $\langle A, \emptyset\rangle$ has the unique stable extension $\operatorname{Th}(A)$, and hence $A=\varphi$ if and only if $(\langle A, \emptyset\rangle, \varphi) \in \operatorname{CRED}(B)$. Therefore, $\operatorname{IMP}(B) \leq_{\mathrm{cd}} \operatorname{CRED}(B)$, and the claim follows with Theorem 3.1.

For the fourth part, it suffices to prove NP-hardness for $\mathrm{N}_{2} \subseteq[B]$. For $\mathrm{L}_{0} \subseteq[B]$, the claim then follows by Lemma 5.1. For $\mathrm{N}_{2} \subseteq[B]$, we obtain NPhardness of $\operatorname{CRED}(B)$ by adjusting the reduction given in the proof of item 3 of Theorem 5.4. Consider the mapping $\varphi \mapsto\left(\left\langle\{\psi\}, D_{\varphi}\right\rangle, \psi\right)$, where $D_{\varphi}$ is the set of default rules constructed from $\varphi$ in Theorem 5.4, and $\psi$ is a satisfiable $B$-formula such that $\varphi$ and $\psi$ do not use common variables. By Theorem 5.4, $\varphi \in$ 3SAT if and only if $\left\langle\{\psi\}, D_{\varphi}\right\rangle$ has a stable extension. As any extension of $\left\langle\{\psi\}, D_{\varphi}\right\rangle$ contains $\psi$, we obtain $3 \mathrm{SAT} \leq_{\mathrm{cd}} \operatorname{CRED}(B)$ via the above reduction.

For the fifth part, the cases $\mathrm{E}_{2} \subseteq[B]$ and $\mathrm{V}_{2} \subseteq[B]$ follow similarly from Lemmas 5.1 and 5.7. It hence suffices to prove the P-hardness for $[B] \in\left\{\mathrm{L}_{1}, \mathrm{~L}_{2}\right\}$ We again provide a reduction from HGAP restricted to hypergraphs whose edges contain at most two source nodes. To this end, we transform a given instance $(H, S, t)$ with $H=(V, F)$, to the $\operatorname{CRED}(\{x \oplus y \oplus z, 1\})$-instance $(\langle W, D\rangle, \varphi)$, where

$$
\begin{aligned}
W:= & \left\{p_{s} \mid s \in S\right\}, \\
D:= & \left\{\frac{p_{\operatorname{src}(e)}: 1}{p_{\operatorname{dest}(e)}}|e \in F,| \operatorname{src}(e) \mid=1\right\} \cup \\
& \left\{\frac{p_{\operatorname{src}_{1}(e)}: 1}{p_{e}}, \frac{p_{\operatorname{src}_{2}(e)}: 1}{p_{e}}, \frac{\left.p_{\operatorname{src}_{1}(e) \oplus p_{\operatorname{src}_{2}(e)} \oplus p_{e}: 1}|e \in F,| \operatorname{src}(e) \mid=2\right\},}{p_{\operatorname{dest}(e)}} \mid\right. \\
\varphi:= & p_{t},
\end{aligned}
$$

and $\left\{\operatorname{src}_{1}(e), \operatorname{src}_{2}(e)\right\}$ denote the source nodes of $e$. As for the correctness, observe that if for some $e \in F$ with $|\operatorname{src}(e)|=2$ both variables $p_{\operatorname{src}_{1}(e)}$ and $p_{\operatorname{src}_{2}(e)}$ can be derived from the stable extension of $\langle W, D\rangle$, then $p_{e}$ and consequently $p_{\text {dest }(e)}$ can be derived. Conversely, if $\operatorname{src}_{1}(e)$ or $\operatorname{src}_{2}(e)$ cannot be derived, then either none or two of the propositions in $p_{\operatorname{src}_{1}(e)} \oplus p_{\operatorname{src}_{2}(e)} \oplus p_{e}$ are satisfied. Thus $p_{\text {dest }(e)}$ cannot be derived from the defaults corresponding to $e$.

Finally, it remains to show NL-hardness for $\mathrm{I}_{2} \subseteq[B]$. We give a $\leq_{\mathrm{cd}^{-}}$ reduction from GAP to $\operatorname{CRED}(\{$ id $\})$. For a directed graph $G=(V, F)$ and two nodes $s, t \in V$, we transform the GAP-instance $(G, s, t)$ with $G=(V, F)$ to the $\operatorname{CRED}\left(\mathrm{I}_{2}\right)$-instance

$$
W:=\left\{p_{s}\right\}, D:=\left\{\frac{p_{u}: p_{u}}{p_{v}} \mid(u, v) \in F\right\}, \varphi:=p_{t} .
$$

Clearly, $(G, s, t) \in$ GAP if and only if $\varphi$ is contained in all stable extensions of $\langle W, D\rangle$. 
This completes the proof of Theorem 5.9.

We will next classify the complexity of the skeptical reasoning problem. The analysis as well as the result are similar to the classification of the credulous reasoning problem (cf. also Fig. 2).

Theorem 5.12. Let $B$ be a finite set of Boolean functions. Then $\operatorname{SKEP}(B)$ is

1. $\Pi_{2}^{\mathrm{p}}$-complete if $\mathrm{S}_{1} \subseteq[B] \subseteq \mathrm{BF}$ or $\mathrm{D} \subseteq[B] \subseteq \mathrm{BF}$,

2. $\Delta_{2}^{\mathrm{p}}$-complete if $\mathrm{S}_{11} \subseteq[B] \subseteq \mathrm{M}$,

3. coNP-complete if $X \subseteq[B] \subseteq Y$, where $X \in\left\{\mathrm{S}_{00}, \mathrm{~S}_{10}, \mathrm{~N}_{2}, \mathrm{~L}_{0}\right\}$ and $Y \in$ $\left\{R_{1}, M, L\right\}$,

4. $\mathrm{P}$-complete if $\mathrm{V}_{2} \subseteq[B] \subseteq \mathrm{V}, \mathrm{E}_{2} \subseteq[B] \subseteq \mathrm{E}$ or $[B] \in\left\{\mathrm{L}_{1}, \mathrm{~L}_{2}\right\}$, and

5. NL-complete if $\mathrm{I}_{2} \subseteq[B] \subseteq \mathrm{I}$.

Proof. The first part again follows from Theorem 4.4 and Lemma 5.1.

For $[B] \in\left\{\mathrm{N}, \mathrm{N}_{2}, \mathrm{~L}, \mathrm{~L}_{0}, \mathrm{~L}_{3}\right\}$, we guess similarly as in Theorem 5.4 a set $G$ of defaults and then verify in the same way whether $W$ and $G$ generate a stable extension $E$. If not, then we accept. Otherwise, we check if $E \models \varphi$ and answer according to this test. This yields a coNP-algorithm for $\operatorname{SKEP}(B)$. Hardness for coNP is achieved by modifying the reduction from Theorem 5.4 (cf. also the proof of Proposition 5.11): map $\varphi$ to $\left(\left\langle\emptyset, D_{\varphi}\right\rangle, \psi\right)$, where $D_{\varphi}$ is defined as in the proof of Theorem 5.4, and $\psi$ is a $B$-formula such that $\varphi$ and $\psi$ do not share variables. Then $\varphi \notin 3 \mathrm{SAT}$ if and only if $\left\langle\emptyset, D_{\varphi}\right\rangle$ does not have a stable extension. The latter is true if and only if $\psi$ is in all extensions of $\left\langle\emptyset, D_{\varphi}\right\rangle$. Hence $\overline{3 \mathrm{SAT}} \leq_{\mathrm{cd}} \operatorname{SKEP}(B)$, establishing the claim.

For all remaining clones $B$, observe that $[B] \subseteq \mathrm{R}_{1}$ or $[B] \subseteq \mathrm{M}$. Hence, Corollary 5.3 and Theorem 5.9 imply the claim.

\section{Conclusion}

In this paper we provided a complete classification of the complexity of the main reasoning problems for default propositional logic, one of the most common frameworks for nonmonotonic reasoning. The complexity of the extension existence problem shows an interesting similarity to the complexity of the satisfiability problem [Lew79], because in both cases the hardest instances lie above the clone $S_{1}$ (with the exception that instances from $D$ are still hard for EXT, but easy for SAT). The complexity of the membership problems, i.e., credulous and skeptical reasoning, rests on two sources: first, whether there exists a unique extension (cf. Lemma 5.2), and second, how hard it is to test for formula implication. For this reason, we also classified the complexity of the implication problem $\operatorname{IMP}(B)$.

A different complexity classification of reasoning for default logic has been undertaken in [CHS07]. In that paper, the language of existentially quantified propositional logic was restricted to so called conjunctive queries, i.e., existentially quantified formulae in conjunctive normal-form with generalized clauses. The complexity of the reasoning tasks was determined depending on the type of clauses that are allowed. We want to remark that though our approach at first sight seems to be more general (since we do not restrict our formulae to CNF), 
the results in [CHS07] do not follow from the results presented here (and vice versa, our results do not follow from theirs).

In the light of our present contribution, it is interesting to remark that by results of Konolige, Gottlob, and Janhunen [Kon88,Got95b,Jan99], propositional default logic and Moore's autoepistemic logic are essentially equivalent. Even more, the translations are efficiently computable. Unfortunately, all of them require a complete set of Boolean connectives, whence our results do not immediately transfer to autoepistemic logic. It is nevertheless interesting to ask whether the exchange of default rules with the introspective operator $L$ yields hitherto unclassified fragments of autoepistemic logic that allow for efficient stable expansion testing and reasoning.

\section{Acknowledgements}

We thank Ilka Schnoor for sending us a manuscript with the proof of the NPhardness of $\operatorname{EXT}(B)$ for all $B$ such that $\mathrm{N}_{2} \subseteq[B]$. We also acknowledge helpful discussions on various topics of this paper with Peter Lohmann.

\section{References}

[BCRV03] E. Böhler, N. Creignou, S. Reith, and H. Vollmer. Playing with Boolean blocks, part I: Post's lattice with applications to complexity theory. SIGACT News, 34(4):38-52, 2003.

[BEZ02] R. Ben-Eliyahu-Zohary. Yet some more complexity results for default logic. Artificial Intelligence, 139(1):1-20, 2002.

[BMTV09a] O. Beyersdorff, A. Meier, M. Thomas, and H. Vollmer. The complexity of propositional implication. Information Processing Letters, 109(18):1071-1077, 2009.

[BMTV09b] O. Beyersdorff, A. Meier, M. Thomas, and H. Vollmer. The complexity of reasoning for fragments of default logic. In Proc. 12th International Conference on Theory and Applications of Satisfiability Testing, volume 5584 of Lecture Notes in Computer Science, pages 51 - 64. Springer Verlag, 2009.

[BO02] P. A. Bonatti and N. Olivetti. Sequent calculi for propositional nonmonotonic logics. ACM Transactions on Computational Logic, 3(2):226-278, 2002.

[CHS07] P. Chapdelaine, M. Hermann, and I. Schnoor. Complexity of default logic on generalized conjunctive queries. In Proc. 9th International Conference on Logic Programming and Nonmonotonic Reasoning, volume 4483 of Lecture Notes in Computer Science, pages 58-70. Springer Verlag, 2007.

[Got92] G. Gottlob. Complexity results for nonmonotonic logics. Journal of Logic Computation, 2(3):397-425, 1992.

[Got95a] G. Gottlob. NP trees and Carnap's modal logic. Journal of the ACM, 42(2):421457, 1995.

[Got95b] G. Gottlob. Translating default logic into standard autoepistemic logic. Journal of the ACM, 42(4):711-740, 1995.

[Jan99] T. Janhunen. On the intertranslatability of non-monotonic logics. Annals of Mathematics and Artificial Intelligence, 27(1-4):79-128, 1999.

[Kon88] K. Konolige. On the relation between default and autoepistemic logic. Artificial Intelligence, 35(3):343-382, 1988. Erratum: Artificial Intelligence, 41(1):115.

[KS91] H. A. Kautz and B. Selman. Hard problems for simple default logics. Artificial Intelligence, 49:243-279, 1991.

[Lew79] H. Lewis. Satisfiability problems for propositional calculi. Mathematical Systems Theory, 13:45-53, 1979.

[McC80] J. McCarthy. Circumscription - a form of non-monotonic reasoning. Artificial Intelligence, 13:27-39, 1980. 
[MT93] V. W. Marek and M. Truszczyński. Nonmonotonic Logic. Artificial Intelligence. Springer Verlag, Berlin Heidelberg, 1993.

[Pap94] C. H. Papadimitriou. Computational Complexity. Addison-Wesley, Reading, MA, 1994.

[Pip97] N. Pippenger. Theories of Computability. Cambridge University Press, Cambridge, 1997.

[Pos41] E. Post. The two-valued iterative systems of mathematical logic. Annals of Mathematical Studies, 5:1-122, 1941.

[Rei80] R. Reiter. A logic for default reasoning. Artificial Intelligence, 13:81-132, 1980.

[SI90] R. Sridhar and S. Iyengar. Efficient parallel algorithms for functional dependency manipulations. In Proc. 2nd International Symposium on Databases in Parallel and Distributed Systems, pages 126-137. ACM, 1990.

[Sti90] J. Stillman. It's not my default: The complexity of membership problems in restricted propositional default logics. In Proc. 8th Conference on Artificial Intelligence, pages 571-578. ACM, 1990.

[Vol99] H. Vollmer. Introduction to Circuit Complexity - A Uniform Approach. Texts in Theoretical Computer Science. Springer Verlag, Berlin Heidelberg, 1999. 